\title{
Efeitos do extrato supercrítico de Melia azedarach (Meliaceae) no controle de Sitophilus zeamais Motschulsky, 1855 (Coleoptera, Curculionidae)
}

\author{
Effects of supercritical extract of Melia azedarach (Meliaceae) on the control of Sitophilus zeamais \\ Motschulsky, 1855 (Coleoptera, Curculionidae)
}

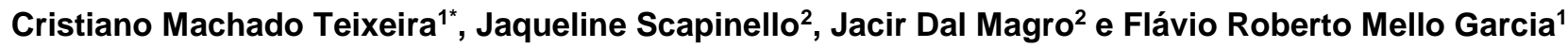 \\ ${ }^{1}$ Universidade Federal de Pelotas, Pelotas, RS, Brasil. *Autor para correspondência: crisakst@yahoo.com.br. \\ ²Universidade Comunitária da Região de Chapecó, Chapecó, SC, Brasil.
}

\begin{abstract}
RESUMO
O presente estudo teve como objetivo testar a eficiência do extrato supercrítico de Melia azedarach no controle de Sitophilus zeamais por meio do contato tarsal. Observou-se a sobrevivência dos insetos a cada $24 \mathrm{~h}$ após o início do experimento. Constatou-se que houve maior mortalidade de insetos nas primeiras $72 \mathrm{~h}$. $\mathrm{O}$ tratamento que teve melhor desempenho considerando-se a relação tempo de ação e número de insetos mortos foi o de concentração $200 \mu \mathrm{L} \mathrm{mL}^{-1}$. A concentração letal média que mata $50 \%$ da população (CL50) obtida após $96 \mathrm{~h}$ do tratamento foi de $165,52 \mu \mathrm{L} \mathrm{mL}^{-1}$, indicando que a maior dose pode ser reduzida com resultados satisfatórios para as condições empregadas no estudo. $O$ extrato de $M$. azedarach revelou-se efetivo no controle de $S$. zeamais em condições de laboratório.
\end{abstract}

PALAVRAS-CHAVE: bioinseticida, cinamomo, contato tarsal, gorgulho-do-milho.

\begin{abstract}
This study aimed to test the efficiency of the Melia azedarach supercritical extract in controlling Sitophilus zeamais through tarsal contact. The survival of insects was observed every $24 \mathrm{~h}$ after the experiment started. A higher mortality of insects was found during the first $72 \mathrm{~h}$. The treatment that had the best performance considering the action time and the number of dead insects was that at $200 \mu \mathrm{L} \mathrm{mL}-1$ concentration. The mean lethal concentration that kills $50 \%$ of the population (LC50) obtained after $96 \mathrm{~h}$ treatment was $165.52 \mu \mathrm{L} \mathrm{ml}-1$, showing that the higher dose can be reduced with satisfactory results for the conditions used in this study. The extract of M. azedarach was effective in controlling S. zeamais under laboratory conditions.
\end{abstract}

KEYWORDS: biopesticides, cinnamon, tarsal contact, maize weevil.

O gorgulho-do-milho, Sitophilus zeamais Motschulsky, 1855 (Coleoptera, Curculionidae), está entre os insetos praga mais importantes de produtos armazenados. Infesta todos os tipos de grãos de cereais e seus produtos derivados. A ação do inseto gera redução de peso, depreciação do valor comercial, redução do valor nutricional, dissemina fungos e favorece a deterioração do produto em alimentos estocados (SULEIMAN et al. 2014, SANTOS et al. 2015, ZAKKA et al. 2015).

Para controle desses insetos utilizam-se inseticidas sintéticos, mas que pelo uso frequente e indiscriminado, acarretam altos níveis de resíduos tóxicos nos alimentos e no meio ambiente, contribuindo com efeitos ambientais e ecológicos como linhagens resistentes de insetos. A utilização de inseticidas menos nocivos ao meio ambiente é uma alternativa sempre bem recebida. Nesse contexto é importante a informação de que plantas são ricas em substâncias do metabolismo secundário úteis no desenvolvimento de métodos seguros aplicados ao controle de insetos, desse modo, os aleloquímicos despontam como possíveis substitutos aos compostos sintéticos com eficiência e baixa toxicidade ao homem e ao meio ambiente (KNAAK \& FIUZA 2010, SOUZA et al. 2015).

A utilização de inseticidas botânicos como uma estratégia dentro do Manejo Integrado de Pragas (MIP) é promissora, principalmente por meio da utilização do Nim, todavia, as pesquisas com outros 
extratos e óleos essenciais devem ser ampliadas (MARANGONI et al. 2012).Várias famílias botânicas apresentam propriedades inseticidas em seus compostos, tais como Myrtaceae (MIGLIORINI et al. 2010, JAIROCE et al. 2016), Lauraceae, Myristicaceae (MIGLIORINI et al. 2010), Rutaceae, Poaceae, Asteraceae, Boraginaceae, Malvaceae, Phaseaceae, Chenopodiaceae, Brassicaceae, Phytolaccaceae, Zingiberaceae (KNAAK et al. 2012), Meliaceae (MIGLIORINI et al. 2010, DEQUECH et al. 2011) e Lamiaceae (MIGLIORINI et al. 2010, PRADO et al. 2013, SAVARIS et al. 2014), Apiaceae, Apoyinaceae, Euphorbiaceae, Fabaceae, Piperaceae, Rutaceae e Solanaceae (MIGLIORINI et al. 2010, LOVATTO et al. 2012).

Muitos trabalhos têm sido realizados para o controle de insetos utilizando pós e extratos de cinamomo (Melia azedarach L. - Meliaceae). No estudo com extrato de folhas de M. azedarach no controle de $S$. zeamais, utilizando diferentes concentrações, foi encontrada uma resposta de $33,3 \%$ de mortalidade em $96 \mathrm{~h}$ (YOHANNES et al. 2014). Em experimento semelhante, foi observado em $24 \mathrm{~h}$ de exposição valores de mortalidade inicial de $16 \%$, e aos sete dias de tratamento o percentual aumentou para $90 \%$ (MARTíNEZCAÑAVERAL et al. 2012). Do mesmo modo, em outro trabalho foi reportada mortalidade de $48,24 \%$ em $192 \mathrm{~h}$ (CHÁVEZ et al. 2010).

Estudos realizados com o extrato de frutos de cinamomo obtidos por meio do solvente dióxido de carbono supercrítico quando adicionado a dieta artificial das larvas mostrou atividade inseticida $\left(\mathrm{DL}_{50}=\right.$ $376,74 \mathrm{mg} \mathrm{kg}^{-1}$ ) e inibidora de crescimento (na concentração de $100 \mathrm{mg} \mathrm{kg}^{-1}$ ) sobre a lagarta do cartucho do milho (SCAPINELLO et al. 2014a, 2014b).

Foram obtidos $78 \%$ de mortalidade de Sitophilus oryzae (L., 1763) em trabalho onde os insetos foram submetidos ao extrato de cinamomo em 10 dias de tratamento (SALJOQI et al. 2006). Utilizando o pó de folhas contra $S$. oryzae, pesquisadores observaram uma mortalidade de $80,54 \%$ em 35 dias (DEVI et al. 2014). Também em experimento com S. zeamais foram encontrados resultados de $83,3 \%$ e $86,6 \%$ de mortalidade em diferentes concentrações após cinco dias de tratamento (YOHANNES et al. 2014).

Utilizando vapor do óleo da semente de cinamomo, outros pesquisadores obtiveram resultados de mortalidade para S. oryzae de 53\% em $168 \mathrm{~h}$ (SARAÇ et al. 2004). Estes estudos evidenciam o potencial inseticida dos extratos de cinamomo, sejam eles brutos, aquosos, alcoólicos e demais formas de obtenção.

Nesse sentido, visando ampliar o rol de metodologias empregadas para o controle alternativo do gorgulho-do-milho, este estudo tem o objetivo de testar o potencial do extrato supercrítico de frutos do cinamomo contra $S$. zeamais, por meio do contato tarsal em condições de laboratório.

As amostras de frutos de Melia azedarach foram coletadas durante o verão (fevereiro), quando a sua coloração é verde e epiderme lisa, na cidade de Chapecó, localizada no sul do Brasil $\left(27^{\circ} 0,5^{\prime} 38^{\prime \prime} \mathrm{S}\right.$, $52^{\circ} 40^{\prime} 0,5^{\prime \prime}$ ). Parte da amostra foi depositada no Herbário da Universidade Comunitária de Chapecó (Brasil) com o número UNO 2841. Após a coleta, os frutos foram secos a $40{ }^{\circ} \mathrm{C}$ durante $48 \mathrm{~h}$ em estufa, e então triturados em liquidificador industrial até a gramatura de $0,85 \mathrm{~mm}$. As amostras foram então armazenadas à temperatura ambiente sob atmosfera de nitrogênio, até o momento da extração. $O$ dióxido de carbono utilizado possuía pureza de 99,9\% na fase líquida.

O aparelho de extração e o procedimento experimental foram descritos em detalhe por SCAPINELLO et al. (2014a). Os experimentos foram realizados em uma unidade de escala laboratorial, o qual consiste de um reservatório de $\mathrm{CO}_{2}$, dois banhos termostáticos, uma bomba de seringa (ISCO 260D), um recipiente de extração com 0,518 L, encamisado, um transdutor de pressão absoluta (Smar, LD301) equipado com um programador portátil (Smar, HT 201) com uma precisão de $\pm 0,12$ bar, um vaso coletor com um tubo de vidro e uma câmara de frio.

Valores de 70,03 $\pm 0,09 \mathrm{~g}$ de frutos secos e triturados foram carregados para dentro do vaso de extração. Em seguida, o $\mathrm{CO}_{2}$ foi bombeado para dentro do leito (pressão de 200 bar), que foi realizada por dois discos de arame de malha $300 \mathrm{em}$ ambas as extremidades. O solvente foi mantido em contato com a amostra durante 30 minutos, para permitir a estabilização do sistema na condição do experimento. Posteriormente, o extrato foi recolhido pela abertura da válvula de microdosagem. Os experimentos foram realizados na temperatura de $60{ }^{\circ} \mathrm{C}$ e pressão de $250 \mathrm{bar}$, usando uma taxa de fluxo de $\mathrm{CO}_{2}$ de $2 \mathrm{~g} \mathrm{~min}^{-1}$ durante $2 \mathrm{~h}$.

A coleta foi realizada em silos de armazenamento de grãos de uma indústria alimentícia no município de Pelotas (3146'19" S 52²0'34" O), Rio Grande do Sul, Brasil. Em laboratório, depois de triados, os insetos foram colocados em potes de vidro cobertos com voal e contendo $400 \mathrm{~g}$ de milho com $13 \%$ de umidade para evitar o desenvolvimento de fungos possivelmente entomopatogênicos que pudessem comprometer a saúde dos insetos e gerar falsos resultados na mortalidade daqueles submetidos aos tratamentos.

Os insetos foram mantidos nos potes por um período de 10 dias e após ocorrer a oviposição nos 
grãos foram retirados e aguardou-se a emergência dos adultos da próxima geração os quais foram selecionados no mesmo dia da emergência, a fim de que tivessem a mesma idade (seis dias de idade) no início do experimento não sendo os resultados deste trabalho influenciados pela senescência dos indivíduos. A triagem, criação e experimentos ocorreram nas condições de temperatura a $25 \pm 2{ }^{\circ} \mathrm{C}$, U.R. 70 $\pm 10 \%$ e fotofase de $12 \mathrm{~h}$, todas as etapas foram realizadas no Laboratório de Ecologia de Insetos da Universidade Federal de Pelotas, Pelotas, RS. A confirmação da espécie foi realizada por meio da terminália dos insetos machos.

A metodologia empregada baseia-se no contato dos tarsos do inseto com o papel-filtro impregnado com extrato segundo metodologia adotada por SALMERON \& OMOTO (2004). Cada unidade experimental foi constituída de uma placa de Petri com 10 insetos não sexados e cada tratamento foi testado com quatro repetições, sendo seis com extrato de $M$. azedarach nas concentrações de $200 \mu \mathrm{L} \mathrm{mL}^{-1}$ (ES-200), $160 \mu \mathrm{L}$ $\mathrm{mL}^{-1}$ (ES-160), $120 \mu \mathrm{L} \mathrm{mL}^{-1}$ (ES-120), $80 \mu \mathrm{LL}^{-1}$ (Es-80), $40 \mu \mathrm{LL}^{-1}$ (ES-40), $20 \mu \mathrm{LL}^{-1}$ (ES-20) e uma testemunha (água destilada), ( $T=25 \pm 2{ }^{\circ} \mathrm{C}, \mathrm{U} . \mathrm{R} .=70 \pm 10 \%$ e fotofase $12 \mathrm{~h}$ ).

Foi dado delineamento inteiramente casualizado (DIC) em esquema fatorial. O experimento teve duração de $96 \mathrm{~h}$. Aplicou-se ANOVA sobre o número de insetos vivos e as médias foram comparadas pelo teste de Tukey $(p<0,05)$. A eficiência dos tratamentos foi calculada pela equação de ABBOTT (1925). Foi também calculada a concentração letal média que mata $50 \%$ da população $\left(C_{50}\right)$ através da correlação entre as concentrações e as mortalidades acumuladas de $S$. zeamais 96 h após o início do experimento.

Visando verificar se os insetos realmente estavam mortos e não se encontravam em tanatose (GAIGER \& VANIN 2006) fez-se um trabalho piloto previamente e observou-se a forma como os insetos suspeitos de estarem mortos ficavam, sendo sinais positivos constatados o recurvamento do rostrum para baixo, as antenas aleatoriamente dispostas em relação ao rostrum, as pernas aleatoriamente dispostas de forma que a porção distal ficava afastada do corpo, ora esticadas ora dobradas, mas nunca unidas ao corpo de forma alinhada, em alguns casos o élitro levemente levantado sendo possível observar parte da asa membranosa.

Houve maior mortalidade de insetos após as $72 \mathrm{~h}$ da aplicação dos tratamentos. Foi possível constatar que existe interação significativa entre os fatores concentração do extrato e horas de exposição, com declínio da sobrevivência em relação ao aumento no tempo de exposição (Figura 1).

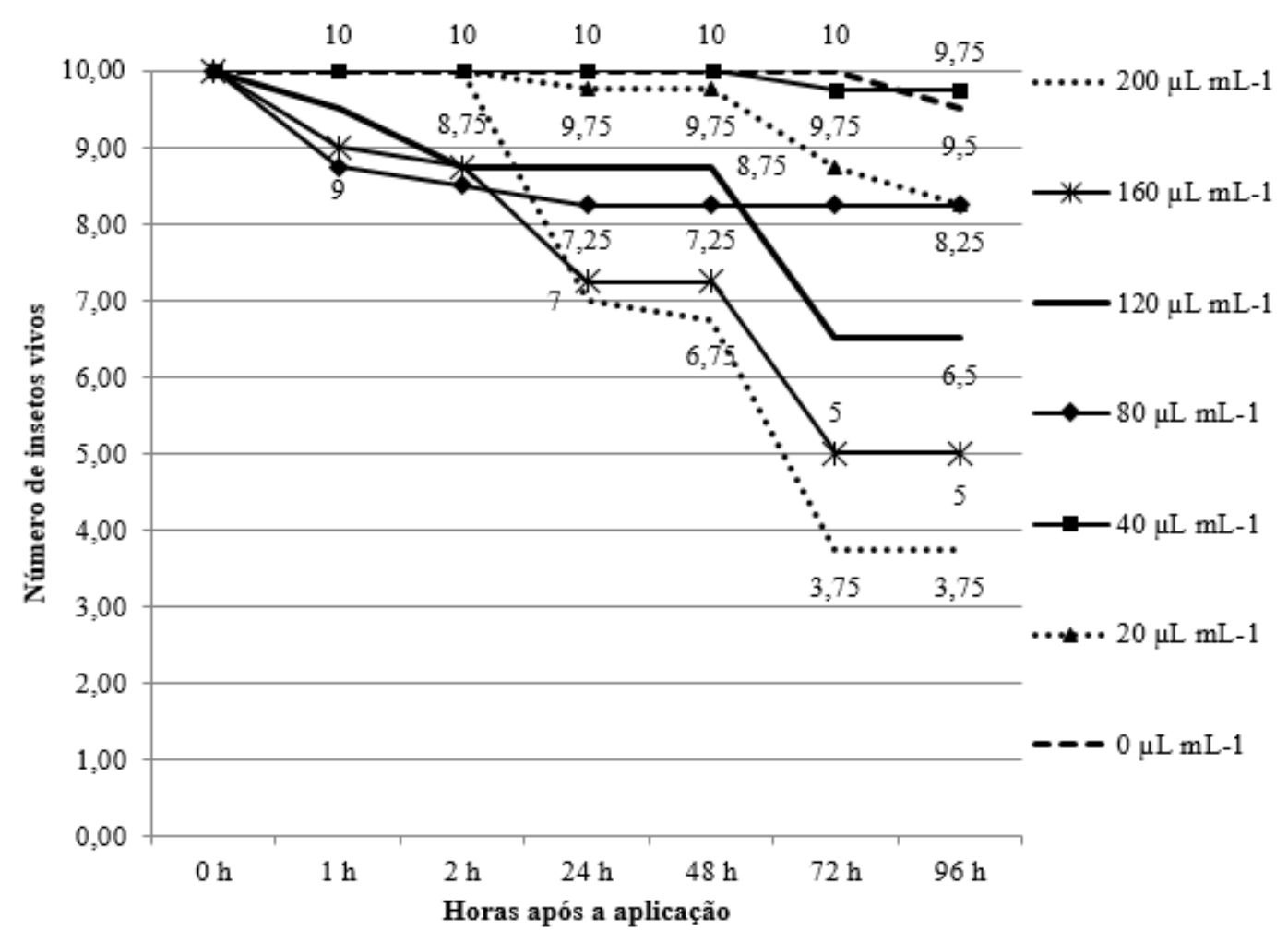

Figura 1. Sobrevivência média dos adultos de Sitophilus zeamais após aplicação dos tratamentos com o extrato supercrítico de frutos de Melia azedarach no decorrer das $96 \mathrm{~h}$ de experimento

Figure 1. Average survival of adults of Sitophilus zeamais after application of treatments with the supercritical extract of fruits of Melia azedarach during the 96 hours of experiment. 
Os tratamentos ES-200, ES-160, ES-120 e ES-80 não apresentaram diferenças significativas entre si, mas diferiram da testemunha (Tabela 1), havendo efeitos dos tratamentos sobre a sobrevivência dos insetos.

Após as duas primeiras horas de aplicação, somente houve diferença no tratamento ES-120. Os tratamentos ES-20, ES-40 e ES-80 não diferiram da testemunha do momento da aplicação até as $48 \mathrm{~h}$ quando se observaram as médias de sobrevivência de 9,$75 ; 10$ e 8,25 , respectivamente.

Ao analisar a $\mathrm{CL}_{50}$, observou-se que a concentração letal está situada entre 141,73 e 189,31 $\mu \mathrm{L} \mathrm{mL}^{-1}$, sendo um valor de $165,52 \mu \mathrm{L} \mathrm{mL}^{-1}$. Observa-se que a partir de $24 \mathrm{~h}$ o extrato botânico demonstrou efeito sobre os insetos. O tratamento que teve maior eficiência foi o ES-200 (62,5\%), seguido por ES-160 (50\%) e ES-120 (35\%).

Tabela 1. Sobrevivência média de Sitophilus zeamais exposto aos tratamentos no decorrer de $96 \mathrm{~h}$ após aplicação do extrato supercrítico de frutos de Melia azedarach em laboratório $\left(T=25 \pm 2{ }^{\circ} \mathrm{C}, \mathrm{U}\right.$. R. $=70 \pm 10 \%$ ).

Table 1. Average survival of Sitophilus zeamais exposed to treatments over $96 \mathrm{~h}$ after application of the supercritical extract of Melia azedarach fruits in the laboratory $\left(T=25 \pm 2{ }^{\circ} \mathrm{C}, \mathrm{R} . \mathrm{H} .=70 \pm 10 \%\right)$.

\begin{tabular}{lll}
\hline Tratamentos $\left(\mu \mathrm{LL}^{-1}\right)$ & Médias $\pm \mathrm{EP}$ & $\mathrm{ET}(\%)$ nas $96 \mathrm{~h}$ \\
\hline ES -200 & $7,32 \pm 0,09 \mathrm{~d}$ & 60,5 \\
ES -160 & $7,46 \pm 0,12 \mathrm{~cd}$ & 47,4 \\
ES -120 & $8,36 \pm 0,11 \mathrm{bcd}$ & 31,6 \\
ES -80 & $8,61 \pm 0,07 \mathrm{bc}$ & 13,2 \\
ES -40 & $9,93 \pm 0,01 \mathrm{a}$ & 2,6 \\
ES -20 & $9,5 \pm 0,03 \mathrm{ab}$ & 13,2 \\
Grupo controle -0 & $9,93 \pm 0,01 \mathrm{a}$ & 0,0 \\
\hline
\end{tabular}

$\mathrm{CV} \%=17,98$

Médias seguidas pela mesma letra não diferem estatisticamente entre si. Tukey $(p<0,05)$. ES=Extrato supercrítico; ET=Eficiência do tratamento (ABBOTT 1925); EP=Erro Padrão.

Os dados do presente estudo mostram que se obteve resultados satisfatórios com as concentrações utilizadas, principalmente as maiores. Outros trabalhos também avaliaram o efeito de $M$. azedarach sobre 0 mesmo inseto ou espécies filogeneticamente relacionadas, porém com a obtenção do extrato por outros métodos e solventes como em meio aquoso (YOHANNES et al. 2014), em meio alcoólico (WONGTONG \& NAWANICH 2001, SALJOQI et al. 2006, MARTÍNEZ-CAÑAVERAL et al. 2012, EL-ARABY et al. 2014) e em meio hexânico (CHÁVEZ et al. 2010, EL-ARABY et al. 2014). Além disso, utilizam folhas para extração em diferentes idades.

No presente estudo utilizando a concentração ES-40 se obteve $2,5 \%$ de eficiência em $72 \mathrm{~h}$. YOHANNES et al. (2014) numa concentração um pouco maior EA-50 (EA = extrato aquoso) encontraram $20 \%$ em 72 h. SALJOQI et al. (2006) obtiveram em 72 h em média 30\% de eficiência com a concentração EE-40 (EE = extrato etanólico). CHÁVEZ et al. (2010) em 192 h observaram uma eficiência de 48,24\% na concentração EH-40 (EH = extrato hexânico) próximo ao obtido por EL-ARABY et al. (2014) em trabalho com Sitophilus granarius (Linnaeus, 1875), por meio de aplicação tópica de EH com a dose letal de $0.2 \mu \mathrm{L}$ $\mathrm{mL}^{-1}$ após $24 \mathrm{~h}$.

Na concentração ES-20 se verificou 12,5\% de eficiência em 72 h e 13,1\% em 96 h. CHÁVEZ et al. (2010) em 192 h registraram 32,4\% de eficiência na mesma concentração. Nas concentrações ES-80 e ES120 foi possível verificar 17,5 e 35\% de eficiência, respectivamente, no período de 72 h e em 96 h se obteve 13,1 e $31,6 \%$. YOHANNES et al. (2014) na concentração intermediária EA-100 obtiveram $23,3 \%$ para o período de $72 \mathrm{~h}$ e $26,6 \%$ em $96 \mathrm{~h}$.

Nas concentrações ES-160 e ES-200 constatou-se eficiência de 50 e 62,5\%, respectivamente, em 72 $\mathrm{h}$, sendo os maiores valores registrados neste trabalho para cada concentração. Em $96 \mathrm{~h}$ se observou mortalidade de 47,3 e $60,5 \%$. YOHANNES et al. (2014) na concentração EA-200 registraram valores de $20 \%$ em 72 h e $23,3 \%$ em 96 h.

Utilizando concentrações muito maiores de extrato, MARTÍNEZ-CAÑAVERAL et al. (2012) obtiveram 
em 24 h em média 2,83\% de eficiência na concentração EE-1000, e em sete dias de exposição registraram uma média de $45,33 \%$ de eficiência.

Cada experimento realizado por estes autores teve tempo de duração diferente dos demais, além disso há grande discrepância nos percentuais de eficiência de um estudo para o outro, havendo resultados para curto período analisado, como o trabalho de MARTíNEZ-CAÑAVERAL et al. (2012) com 16\% em $24 \mathrm{~h}$ e de longa duração com 78\% em 10 dias (SALJOQI et al. 2006) e 98,7\% em sete dias (MARTíNEZCAÑAVERAL et al. 2012). CHÁVEZ et al. (2010), encontraram uma mortalidade de $9,06 \%$ em $96 \mathrm{~h}$ e $48,24 \%$ em $192 \mathrm{~h}$.

Os resultados deste estudo comparados com os demais trabalhos realizados com extratos de cinamomo (WONGTONG \& NAWANICH 2001, SALJOQI et al. 2006, CHÁVEZ et al. 2010, MARTíNEZCAÑAVERAL et al. 2012, YOHANNES et al. 2014) indicam que pode haver uma grande variação na eficiência dos extratos devido a fatores como parte da planta utilizada, método de extração, solvente utilizado e tempo de exposição. Isso é especialmente notado no trabalho de WONGTONG \& NAWANICH (2001) em que os autores embora utilizassem concentrações altas não obtiveram resultados satisfatórios na mortalidade dos insetos.

O cinamomo não é empregado apenas na forma de extratos aquosos, hexânico ou etanólico, alguns autores utilizam pós de folhas ou outras partes da planta no controle dos gorgulhos $S$. zeamais e $S$. oryzae (DEFAGÓ et al. 2009, ESPINOZA et al. 2012, CRUZ et al. 2013, DEVI et al. 2014).

Além dos resultados obtidos com os curculionídeos, outros trabalhos utilizando extratos de cinamomo contra diferentes insetos e ácaros veem sendo realizados (MIGLIORINI et al. 2010 MANZOOR et al. 2011, KOSMA et al. 2014, BUSATO et al. 2015, SOUZA et al. 2015, AKHTAR et al. 2015) e é inegável a contribuição dos estudos nessa área para o aprimoramento do uso de produtos derivados de cinamomo.

Mesmo com a utilização apenas de pós de folhas e outras partes da planta, muitos estudos têm demonstrado alta eficiência no controle de insetos, é o caso do controle feito com pó de folhas e sementes de cinamomo contra Callosobruchus macullatus (Fabr., 1775) (Coleoptera: Chrysomelidae) no qual se obteve $100 \%$ de mortalidade após $96 \mathrm{~h}$ (KOSMA et al. 2014). Outro inseto do mesmo gênero $C$. chinensis L., 1758, foi fortemente afetado pelos extratos de cinamomo (REHMAN \& KHAN 2015). Em outro trabalho com Diabrotica speciosa (Germar, 1824) (Coleoptera: Chrysomelidae) o efeito do óleo essencial reduziu a população de larvas do mesmo modo que o inseticida fipronil utilizado como um controle no estudo, além disso o efeito inseticida do pó de folhas sobre os insetos foi acima de 80\% (SOUZA et al. 2015) em outro ainda com o mesmo inseto se obteve um percentual de 100\% de mortalidade após $240 \mathrm{~h}$ utilizando extrato bruto (MIGLIORINI et al. 2010).

Em muitos casos os efeitos não letais têm sido observados como redução da capacidade reprodutiva com redução da postura de ovos e progênie com defeitos ou atuando como reguladores de crescimento, além de exercerem atividade antialimentar (SAXENA 1987, VALLADARES et al. 2003, AKHTAR et al. 2015).

O próprio extrato supercrítico já foi utilizado para o controle de Spodoptera frugiperda (J. E. Smith, 1797) (Lepidoptera: Noctuidae) inclusive atuando como inibidor de crescimento e atingindo $100 \%$ de mortalidade e baixa viabilidade pupal, além da emergência de adultos com deformidades em baixas doses (SCAPINELLO et al. 2014a, 2014b).

As perspectivas futuras são de utilizar o extrato supercrítico na indução de efeitos subletais como atividade antialimentar, reguladora de crescimento, repelência e redução da fecundidade, assim como outras alterações comportamentais induzidas nos insetos

O emprego do extrato supercrítico de $M$. azedarach revelou-se efetivo no controle de $S$. zeamais por meio do contato tarsal em condições de laboratório. Nesse estudo foi realizada apenas a primeira etapa que servirá de base para estudos futuros sobre os efeitos do extrato supercrítico na população do gorgulho. Existe espaço para mais estudos com o extrato supercrítico, uma vez que este trabalho não abordou os efeitos subletais sobre a população do inseto alvo.

\section{REFERÊNCIAS}

ABBOTT WS. 1925. A method of computing the effectiveness of an insecticide. Journal of Economic Entomology 18: 265-266.

AKHTAR S et al. 2015. Antifeedant Effect of Essential Oils of Five Indigenous Medicinal Plants Against Stored Grain Insect Pests. Pakistan Journal of Zoology 47: 1045-1050.

BUSATO MA et al. 2015. Potencial larvicida de Melia azedarach L. e Ilex paraguariensis A. St.-Hil. no controle de Aedes aegypti (Linnaeus, 1762) (Diptera: Culicidae). Ciência e Natura 37: 277-282.

CHÁVEZ EC et al. 2010. Evaluación de aceites y extractos vegetales para el control de Sitophilus zeamais y su efecto 
en la calidad de semilla de maíz. Revista de la Facultad de Ciencias Agrarias 42: 135-145.

CRUZ ML et al. 2013. Efectividad de formulados a base de extractos de Nim, Paraíso y Eucalipto para el control Sitophilus oryzae (L). Revista Agroecosistemas 1: 157-164.

DEFAGÓ MT et al. 2009. Melia azedarach extracts: A potential tool for insect pest management. In: GOVIL JN \& SINGH VK (Eds.). Recent Progress in Medicinal Plants. Houston: Studium Press LLC. p.17-33.

DEQUECH STB et al. 2011. Atividade inseticida de extratos de meliáceas sobre Caliothrips phaseoli Hood, 1912 (Thysanoptera: Thripidae) em cultivos em estufa plástica. Revista da Faculdade de Zootecnia, Veterinária e Agronomia 18: 68-77.

DEVI MB et al. 2014. Effects of Six Botanical Plant Powder Extracts on the Control of Rice Weevil, Sitophilus oryzae L. in Stored Rice Grains. International Journal of Agriculture Innovations and Research 2: 683-686.

EL-ARABY RE et al. 2014. Insecticidal properties of some plant extracts against Granary Weevil, Sitophilus granarius L. Journal of Applied Plant Protection 2: 7-11.

ESPINOZA ME et al. 2012. Actividad insecticida de Melia azedarach L. (Meliaceae) sobre Sitophilus zeamais Motschulsky (Coleoptera: Curculionidae). Chilean Journal of Agricultural \& Animal Science 28: 81-87.

GAIGER F \& VANIN SA. 2006. The elytro-femoral stridulatory apparatus in Curculionidae (Coleoptera), with notes on the acoustic behavior of Arniticus hylobioides (Boheman 1843) and Erodiscus proximus (Viana 1959), and thanatosis display in the latter species. Annales de la Société Entomologique de France 42: 165-170.

JAIROCE CF et al. 2016. Insecticide activity of clove essential oil on bean weevil and maize weevil. Revista Brasileira de Engenharia Agrícola e Ambiental 20: 72-77.

KNAAK N \& FIUZA LM. 2010. Potencial dos óleos essenciais de plantas no controle de insetos e microrganismos. Neotropical Biology and Conservation 5: 120-132.

KNAAK N et al. 2012. Atividade inseticida de extratos de plantas medicinais sobre Spodoptera frugiperda (J. E. Smith) (Lepidoptera :Noctuidae). Bioassay 7: 1-6.

KOSMA P et al. 2014. Bioefficacity of the powder of Melia azedarach seeds and leaves against Callosobruchus maculatus, on cowpea seeds (Vigna unguiculata) in storage. Journal of Agricultural Research and Development 5: $72-$ 78.

LOVATTO PB et al. 2012. A interação co-evolutiva entre insetos e plantas como estratégia ao manejo agroecológico em agroecossistemas sustentáveis. Interciencia 37: 657-663.

MANZOOR $\mathrm{F}$ et al. 2011. Effect of ethanolic plant extracts on three storage grain pests of economic importance. Pakistan Journal of Botany 43: 2941-2946.

MARANGONI C et al. 2012. Utilização de óleos essenciais e extratos de plantas no controle de insetos. Revista de Ciências Ambientais 6: 95-112.

MARTÍNEZ-CAÑAVERAL D et al. 2012. Calidad de semilla de maíz tratada con extractos de hojas de Melia azedarach L. contra Sitophilus zeamais Motschulsky em Almacén. Agraria 9: 1-6.

MIGLIORINI P et al. 2010. Eficiência de extratos vegetais no controle de Diabrotica speciosa (Germar, 1824) (Coleoptera: Chrysomelidae), em laboratório. Biotemas 23: 83-89.

PRADO GP et al. 2013. Alphitobius diaperinus (Coleoptera: Tenebrionidae) susceptibility to Cunila angustifolia essential oil. Journal of Medical Entomology 50: 1040-1045.

REHMAN HU \& KHAN SM. 2015. Lethal and sub-lethal effect of bakain seed aqueous extract on the adults of pulse beetle, Callosobruchus chinensis L. (Coleoptera: Bruchidae). International Journal of Entomological Research 3: 1-7.

SALJOQI AUR et al. 2006. Effects of six plant extracts on rice weevil Sitophilus oryzae L. in the stored wheat grains. Journal of Agricultural and Biological Science 1: 1-5.

SALMERON E \& OMOTO C. 2004. Mistura de deltametrina e clorpirifós no manejo da resistência de Blattella germanica (Linnaeus, 1757) (Dictyoptera: Blattellidae) a deltametrina. Entomotropica 19: 85-89.

SANTOS ACV et al. 2015. Use of plant oils from the southwestern Amazon for the control of maize weevil. Journal of Stored Products Research 63: 67-70.

SARAÇ A et al. 2004. The assessment of toxicity of the Melia azedarach seed oil against stored product insects. Integrated Protection of Stored Products IOBC Bulletin/wprs 27: 145-149.

SAVARIS M et al. 2014. Evaluation of extracts and essential oils from "poejo", Cunila angustifolia (Lamiales: Lamiaceae) leaves to control adults of maize weevil, Sitophilus zeamais (Coleoptera: Curculionidae). International Journal of Entomological Research 2: 23-28.

SAXENA RC. 1987. Antifeedants in tropical pest management. International Journal of Tropical Insect Science 8: 731 736.

SCAPINELLO J et al. 2014a. Effects of supercritical $\mathrm{CO}_{2}$ extracts of Melia azedarach $\mathrm{L}$. on the control of fall armyworm (Spodoptera frugiperda). The Journal of Supercritical Fluids 93: 20-26.

SCAPINELLO J et al. 2014b. Insecticidal and growth inhibiting action of the supercritical extracts of Melia azedarach on Spodoptera frugiperda. Revista Brasileira de Engenharia Agricola e Ambiental 18: 866-872.

SOUZA BHS et al. 2015. Formulations of Melia azedarach to control Diabrotica speciosa (Germar) (Coleoptera: Chrysomelidae) larvae in corn and plant enhancement. Neotropical Entomology 44: 173-179.

SULEIMAN R et al. 2014. Is flint corn naturally resistant to Sitophilus zeamais infestation? Journal of Stored Products Research 60:19-24.

VALLADARES G et al. 2003. Actividad antialimentaria e insecticida de un extracto de hojas senescentes de Melia azedarach (Meliaceae). Revista de la Sociedad Entomológica Argentina 62: 53-61. 
WONGTONG S \& NAWANICH S. 2001. Some Insecticidal Plant Extracts for controlling maize weevil, Sitophilus zeamais Motschulsky (Coleoptera: Curculionidae). Kasetsart Journal Natural Science 35: 259-270.

YOHANNES A et al. 2014. Evaluation of certain plant leaf powders and aqueous extracts against maize weevil, Sitophilus zeamais Motsch. (Coleoptera: Curculionidae). Asian Journal of Agricultural Sciences 6: 83-88.

ZAKKA U et al. 2015. Efficacy of combining varietal resistance with harvest time and planting date for the management of Sitophilus zeamais Motschulsky infestation in stored maize. Journal of Stored Products Research 60: 31-35. 